\title{
Characterization of Alginate/Silver Nanobiocomposites Synthesized by Solution Plasma Process and Their Antimicrobial Properties
}

\author{
Sangwoo Nam, ${ }^{1}$ D. MubarakAli, ${ }^{2}$ and Jungwan Kim $^{2}$ \\ ${ }^{1}$ Division of Life Sciences, Incheon National University, Incheon 406-772, Republic of Korea \\ ${ }^{2}$ Division of Bioengineering, Incheon National University, Incheon 406-772, Republic of Korea \\ Correspondence should be addressed to Jungwan Kim; kjw5864@inu.ac.kr
}

Received 14 September 2015; Revised 25 January 2016; Accepted 27 January 2016

Academic Editor: Lavinia Balan

Copyright (c) 2016 Sangwoo Nam et al. This is an open access article distributed under the Creative Commons Attribution License, which permits unrestricted use, distribution, and reproduction in any medium, provided the original work is properly cited.

Solution plasma process (SPP) was adopted to prepare alginate/silver nanoparticle (AL/AgNP) biocomposites. The biocomposites were synthesized in solutions of varying concentrations of $\mathrm{AgNO}_{3}(1-5 \mathrm{mM})$ and alginate $(0.1-0.3 \%$, w/w) by discharging plasma for $7 \mathrm{~min}$ at $800 \mathrm{~V}$ with $30 \mathrm{kHz}$ frequency using a pulsed unipolar power supply. The AL/AgNP emulsion was fabricated into 3D scaffolds by freeze drying and lyophilization and then stabilized by cross-linking via UV irradiation. UV-Vis spectroscopy of the biocomposites showed a characteristic absorbance at the maximum of $415-440 \mathrm{~nm}$ with increase in the intensity of the peaks as the concentration of $\mathrm{AgNO}_{3}$ increased. FE-SEM analysis showed that the 3D scaffolds had microporous structures with fine and uniform pores of 3-9 $\pm 2.0 \mu \mathrm{m}$ in diameter. TEM analysis revealed that AgNPs in the biocomposites were in spherical shape with size range of 5-40 $\pm 2.0 \mathrm{~nm}$ (AL0.3/Ag5) and well distributed in the matrix. The AL/AgNP biocomposites showed microbicidal activity against 9 human pathogens with MIC of $9.6-21 \mu \mathrm{g} / \mathrm{mL}$ for bacteria and $85-425 \mu \mathrm{g} / \mathrm{mL}$ for fungi. Almost all of the E. coli cells $(99.8 \%)$ were killed by the treatment with $42.5 \mu \mathrm{g} / \mathrm{mL}$ of AgNPs at room temperature for $1 \mathrm{~h}$.

\section{Introduction}

There is prodigious research for the development of nanomaterials via facile, reliable, economical, and ecofriendly procedures. Very recently, solution plasma process (SPP) has been proposed as such a technique that would synthesize metal nanoparticles and composites of metal-polymer [1]. SPP involves sequence of physical and chemical reactions, where the water molecules are split into free radicals $\left(\mathrm{H}^{*}, \mathrm{OH}^{*}\right.$, electrons) and solutes (precursors) into ions to nanoparticles during plasma discharge in the solution [2]. The process, therefore, could be applied to synthesis of metal nanoparticles [3] and their simultaneous fabrication into biopolymers [4,5].

Silver nanoparticles (AgNPs) exhibit versatile characteristics such as conductivity, stability, and catalytic and antimicrobial activities among other nanoparticles [6]. The most common method known for the synthesis of AgNPs is chemical reduction of silver salt (usually $\mathrm{AgNO}_{3}$ ) by adding a reducing agent such as sodium borohydrate $\left(\mathrm{NaBH}_{4}\right)$ and citrate [7-9]. Therefore, there has been an urging demand to develop an ecofriendly method for the synthesis of AgNPs to avoid the usage of hazardous chemicals as reducing and stabilizing agents. Silver based composites as well as silver element itself have been reported to exhibit antimicrobial activity against a wide range of microorganisms [10].

Previously, gelatin was employed as a matrix to prepare AgNP biocomposites using SPP as an effort to develop wound dressing. However, gelatin derived from collagen, an animal protein, and possibility of viral contamination has become a great concern in using it for biomedical application. On the other hand, alginate is a biopolymer composed of repeating disaccharide subunits of mannuronate ( $\mathrm{M}$ block) and gluronate ( $G$ block), a major component in the cell walls of brown algae [11]. It has been used for versatile medical applications including cell immobilization and drug delivery for its nontoxicity and biocompatibility in organic environments and ease of gelation [12,13]. Biomaterials based on alginate have been particularly known to be effective in 
wound healing. Therefore, alginate has been credited with high likelihood to be suitable as a matrix fabricating AgNPs, thereby forming nanometal-biocomposite. Balavandy et al. [14] have reported synthesis of alginate based AgNPs using $\mathrm{NaOH}$ as reducing and accelerator reagent for potential applications in chemical pharmaceutical and food industries. Gold nanoparticles dispersed in an alginate gel matrix were synthesized by solution plasma sputtering in alginate solution and gold electrodes [15] for the same purposes. However, no biological evaluation has been made by either group.

The purpose of this study was to synthesize alginate based AgNP (AL/AgNP) biocomposites for the development of more effective and safer biomedical materials using SPP, by which size and shape of AgNPs could be controlled in a much simpler and faster way than the conventional chemical processes could offer. The nanoemulsions or 3D scaffolds of alginate embedded with AgNPs were characterized for their physical properties by UV-Vis spectroscopy, transmission electron microscopy (TEM), and field emission scanning electron microscopy (FE-SEM) equipped with an energy dispersive spectrometry (EDS). In addition, antimicrobial and microbicidal activity of the AL/AgNP biocomposites were tested against various pathogenic bacteria and fungi. The results obtained in this study revealed that stable AgNP nanobiocomposites could be formed using alginate (0.1$0.3 \%)$ at much lower concentration than using gelatin (1.0$5.0 \%$ ) by discharging plasma for $7 \mathrm{~min}$ in $\mathrm{AgNO}_{3}$ solutions. The AL/AgNP biocomposites showed higher antibacterial but lower antifungal activity than the gelatin based biocomposites did, suggesting they would be safe to eukaryotes such as human cells.

\section{Materials and Methods}

2.1. Chemicals. Alginate (Sigma-Aldrich, USA) and $\mathrm{AgNO}_{3}$ (Junsei Co., Japan) were used for the synthesis of AL/AgNP biocomposites. Luria-Bertani broth (LB; yeast extract $0.5 \%$, $\mathrm{NaCl} 0.5 \%$, and tryptone $1 \%$ ), potato dextrose broth (PD; potato dextrose $2.4 \%$ ), yeast peptone broth (YPD; yeast extract $1 \%$, peptone $2 \%$, and dextrose $2 \%$ ), and MüellerHinton agar (beef extract $0.2 \%$, acid digest of casein $1.75 \%$, starch $0.15 \%$, and agar $1.7 \%$ ) were purchased from Difco Co. (USA) and used for microbial culture and antimicrobial activity tests.

2.2. Solution Plasma Process Set-Up. Briefly, $300 \mathrm{~mL}$ of solution containing 1, 3, or $5 \mathrm{mM} \mathrm{AgNO}_{3}$ (Junsei Co., Japan) and 0.1 or $0.3 \%(\mathrm{w} / \mathrm{w})$ alginate was placed in a $500 \mathrm{~mL}$ Teflon chamber specially designed for the process under ambient conditions as described previously $[5,16]$. Plasma was generated in the solution using a pulsed field unipolar power supply (IAP-1010, EN Technology, Korea) at 800 voltages with $30 \mathrm{kHz}$ of frequency for $7 \mathrm{~min}$ between two tungsten electrodes placed in tandem with a gap of $1 \mathrm{~mm}$. A magnetic stirrer was used for the constant mixing and complete dispersion of the solutes in the solution. The products of the process were notated as AL $x / \operatorname{Ag} y$, where $x$ and $y$ represent the concentration of alginate and $\mathrm{AgNO}_{3}$, respectively.
2.3. Fabrication and Characterization of AL/AgNP Biocomposites. AL/AgNP biocomposites were fabricated by freeze drying. The nanobiocomposite solutions $(5 \mathrm{~mL}$ each) were transferred into a sterile petri dish and frozen at $-80^{\circ} \mathrm{C}$ overnight in a deep freezer (ULT2586, Thermo Scientific, USA). The frozen samples were lyophilized at $-40^{\circ} \mathrm{C}$ with the pressure of $6.38 \times 10^{-4} \mathrm{MPa}$ using a freeze dryer (FDV7024 , OPERON, Korea). The dried samples were then crosslinked by UV irradiation $(254 \mathrm{~nm})$ for $30 \mathrm{~min}$ to reduce water solubility and enhance stability.

2.4. Characterization of AL/AgNP Biocomposites. Formation of AgNPs was confirmed by UV-Visible spectroscopy (UV3600, UV-Vis NIR spectrophotometer, Shimadzu, Japan) in the range of $180-1,100 \mathrm{~nm}$. Structures of the biocomposites in 3D scaffold form were investigated using FE-SEM (JEOLJSM-7001F, JEOL Ltd., Japan) equipped with an EDS. TEM analysis (JEOL-JSM-2010, acceleration voltage $200 \mathrm{keV}$, JOEL Ltd., Japan) was carried out to examine the size, shape, and crystalline structure of AgNPs in the matrix. Size distribution of AgNPs in the alginate matrix was evaluated by measuring diameters of the particles. Diameters of more than 100 nanoparticles were measured manually according to the scale bar and their size distribution was presented as percentage of the total number of nanoparticles in each size group.

2.5. Antimicrobial Activity Tests of AL/AgNP Biocomposites. Antimicrobial activity of AL/AgNP biocomposites was examined against 9 pathogens of Gram-negative bacteria (Escherichia coli, Vibrio vulnificus, V. parahaemolyticus, Pseudomonas stutzeri, and P. aeruginosa), Gram-positive bacteria (Staphylococcus aureus and Bacillus cereus), a yeast (Candida albicans), and a fungus (Aspergillus parasiticus). The pathogens were cultured under the conditions as described previously [5] and tested for antimicrobial activity as follows.

2.5.1. Agar Diffusion Assay. Antimicrobial activity of AL/AgNP biocomposite scaffolds was assessed by inhibition of microbial growth on agar plates, which appeared as a clear zone formed around the disc of the scaffolds, according to the Kirby-Bauer agar diffusion method [17]. About $10^{4}$ colony forming units (CFUs) of freshly cultured microbial cells or spores were spread on Müeller-Hinton agar plates and discs of the AL0.3/Ag1, AL0.3/Ag3, or AL0.3/Ag5 biocomposite scaffolds (5 $\mathrm{mm}$ in diameter) were placed on them aseptically. All the bacteria were cultured at $37^{\circ} \mathrm{C}, \mathrm{C}$. albicans at $30^{\circ} \mathrm{C}$, and $\mathrm{A}$. parasiticus at $25^{\circ} \mathrm{C}$. The resulting zones of growth inhibition were measured to assess antimicrobial effect in $24 \mathrm{~h}$ (bacteria), $48 \mathrm{~h}$ (yeast), or $72 \mathrm{~h}$ (fungus) of incubation.

2.5.2. Minimal Inhibitory Concentration (MIC) Assay. MIC of the biocomposite (AL0.3/Ag5) required for complete growth inhibition of each microorganism was determined by adding $0.0-21 \mu \mathrm{g} / \mathrm{mL}$ of $\mathrm{AL} / \mathrm{AgNP}$ to each bacterial culture $(2 \times$ $10^{5}$ CFUs; $5 \mathrm{~mL}$ ), $0.0-170 \mu \mathrm{g} / \mathrm{mL}$ for C. albicans, and $0.0-$ $510 \mu \mathrm{g} / \mathrm{mL}$ for $A$. parasiticus. The tubes were incubated at $25-$ $37^{\circ} \mathrm{C}$ for $18-24 \mathrm{~h}$ with gentle shaking. MIC was determined as the lowest concentration of the nanobiocomposite, at which 
no visible microbial growth (no turbidity) was observed in comparison with that of the control tube to which no nanobiocomposites were added. In order to check the fungal growth more accurately, the fungal cultures were filtered through cellulose nitrate membranes (pore size of $0.45 \mu \mathrm{m}$; Sartorius Stedim, Germany) and the filters were incubated on PDA plates at $25^{\circ} \mathrm{C}$ for several days to detect any mycelial growth.

2.5.3. Assay of CFU Reduction. Microbicidal effect of AL/AgNP biocomposites was assessed quantitatively by examining reduction in $\mathrm{CFU}$ by the biocomposites. The amount of silver in AL0.3/Ag1, AL0.3/Ag3, and AL0.3/Ag5 that was added to each culture was equivalent to $8.5,22.5$, and $42.5 \mu \mathrm{g} / \mathrm{mL}$, respectively. A precise amount of each AL/AgNP biocomposite was added to LB or YPD broth inoculated with $\sim 10^{6} \mathrm{CFUs} / \mathrm{mL}$ of bacteria (E. coli, $P$. aeruginosa, $P$. stutzeri, V. vulnificus, V. parahaemolyticus, S. aureus, and $B$. cereus) or yeast (C. albicans) and the tubes were left to stand at room temperature for $1 \mathrm{~h}$. Microbial cultures containing AL0.3/Ag0 were used as controls in all of the experimental sections. In $1 \mathrm{~h}$ of incubation, an aliquot of culture was taken and inoculated on LB or YPD agar plates after appropriate dilution for $16-24 \mathrm{~h}$ at 37 or $30^{\circ} \mathrm{C}$ to determine if $\mathrm{CFU}$ survived the biocomposite treatment.

\section{Result and Discussion}

3.1. Synthesis of AL/AgNP Biocomposites. AL/AgNP biocomposites were effectively synthesized by the one-pot synthesis method of SPP. A total of six different biocomposites were prepared in various combinations of the alginate $(0.1,0.3 \%)$ and $\mathrm{AgNO}_{3}(1,3,5 \mathrm{mM})$ concentrations. In the previous study, 1,3 , or $5 \%$ of gelatin was used as the matrix in preparing biocomposite using SPP. However, alginate could not be used at the concentrations of $0.5 \%$ and above, but only at concentrations of $0.3 \%$ and below due to instability and viscosity of the solutions. The temperature and color of the alginate and $\mathrm{AgNO}_{3}$ solutions before plasma discharge were $28^{\circ} \mathrm{C}$ and light yellow, respectively. Once plasma was discharged in the solutions, the color of the solutions turned to dark brown probably due to AgNPs formation and temperature of the solutions was elevated to $70^{\circ} \mathrm{C}$ during discharge for 7 min (Figure 1; insight). Reduction of silver from ionic into metallic nanoparticles in the alginate matrix was likely to be caused by the free radicals released during plasma discharge. When AL/AgNP biocomposites were synthesized by SPP, discharge time, amounts of the precursor $\left(\mathrm{AgNO}_{3}\right)$, and the matrix (alginate) were contemplated. The color intensity was developed depending on the concentrations of $\mathrm{AgNO}_{3}$ and the polymer in use as well as the discharge time [18]. Even though the synthesis of AgNPs in biopolymer has been reported by numerous research groups $[2,6,8,9]$, significance of the present study was highlighting the efficacy and purity of the synthesized AL/AgNP biocomposite for biomedical applications. Therefore, SPP was proved to be a facile means to generate metallic NPs in a biologically functional polymer matrix within short period without adding perilous chemicals as reducing and stabilizing agent $[4,5,15]$.

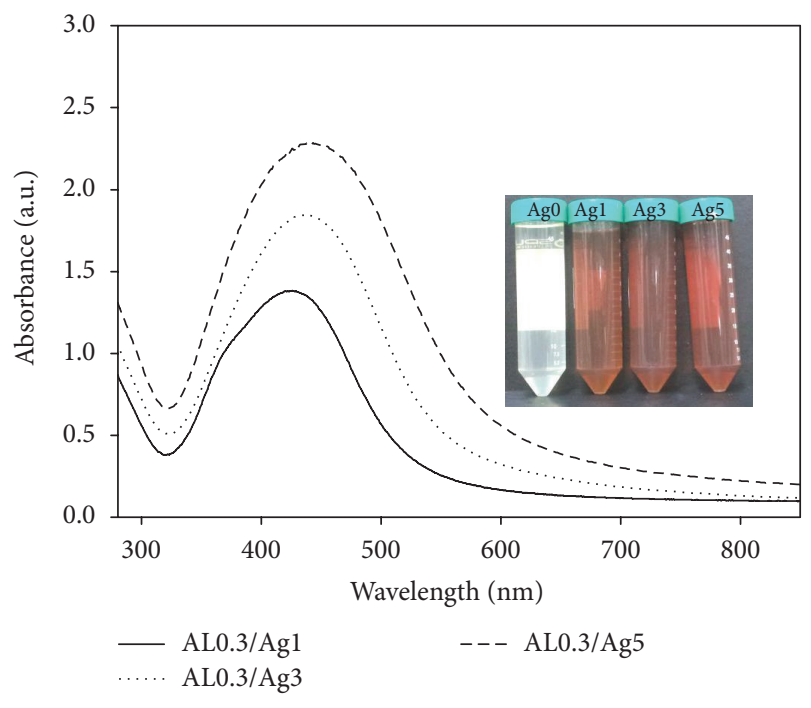

FIgURE 1: UV-Vis spectra of the AL/AgNP biocomposites. The biocomposites, AL0.3/Ag1, AL0.3/Ag3, and AL0.3/Ag5, had surface plasmon resonance at $415-440 \mathrm{~nm}$. The inset picture showed the biocomposites turned to brown during plasma discharge.

Synthesis of AgNPs embedded in the alginate matrix was analyzed using a UV-Visible spectrophotometer (Figure 1). The UV-Visible spectra indicated that AgNPs were formed and the intensity of the peaks was dependent on the concentration of $\mathrm{Ag}$ in the biocomposites. When the concentration of Ag was $1 \mathrm{mM}$, the highest peak was found at $415 \mathrm{~nm}$. As the concentration of $\mathrm{Ag}$ increased to $3 \mathrm{mM}$ and $5 \mathrm{mM}$, the highest peak moved forward to $417 \mathrm{~nm}$ and $440 \mathrm{~nm}$, respectively (Figure 1). The red shift of UV-Visible spectra was observed when the concentration of Ag was increased and suggested that nanoparticles were formed [18]. The UV-Visible spectra of AL0.1/Ag1, AL0.1/Ag3, and AL0.1/Ag5 emulsions synthesized using $0.1 \%$ alginate and various concentrations of $\mathrm{AgNO}_{3}(1$, 3 , and $5 \mathrm{mM}$ ) showed the maximal absorbance at $410 \mathrm{~nm}$, $427 \mathrm{~nm}$, and $433 \mathrm{~nm}$, respectively, and peaks were smaller than those of AL0.3/AgNPs (data not shown).

3.2. Characterization of the AL/AgNP Biocomposites. $3 \mathrm{D}$ scaffolds of the AL/AgNP biocomposites were analyzed using a FE-SEM (Figure 2). Well defined microporous structure with smooth surface was observed in AL0.3/Ag0 and AL0.3/Ag5 (Figures 2(a) and 2(b)). On the other hand, AL0.1/Ag5 showed very irregular porous spongy surface (Figure 2(c)). Microstructures of AL0.3/Ag0, AL0.1/Ag1, and AL0.3/Ag5 had pore sizes in a rather wide range of $2-45 \mu \mathrm{m}$. The pores of AL0.3/Ag0, AL0.1/Ag1, and AL0.3/Ag5 had a mean diameter of $3.5 \pm 0.2,2.5 \pm 0.2$, and $9.7 \pm 0.2 \mu \mathrm{m}$, respectively. The results indicated that porous size increased as the concentration of alginate increased. Cross-sectional view of the microporous AL0.3/Ag5 biocomposite showed a distinct microfibril structure at the edges (Figure 2(d)). Previously, cellulose/AgNP biocomposites also had microporous structures with such microfibrils at the edges [5]. Biocomposites with microfibril structures could be used as the scaffolds for cell proliferation in tissue engineering application [18] and the development 


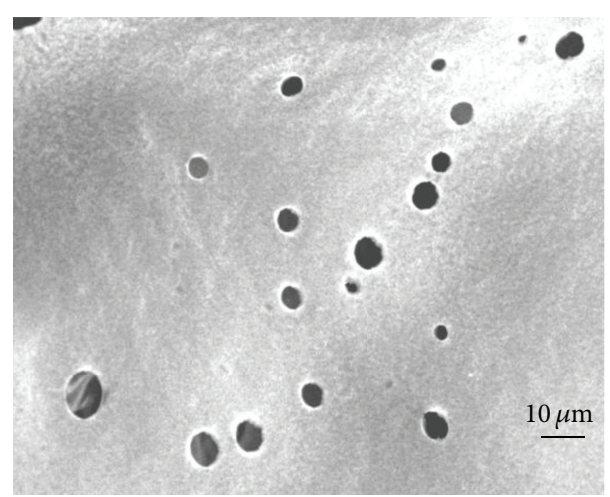

(a)

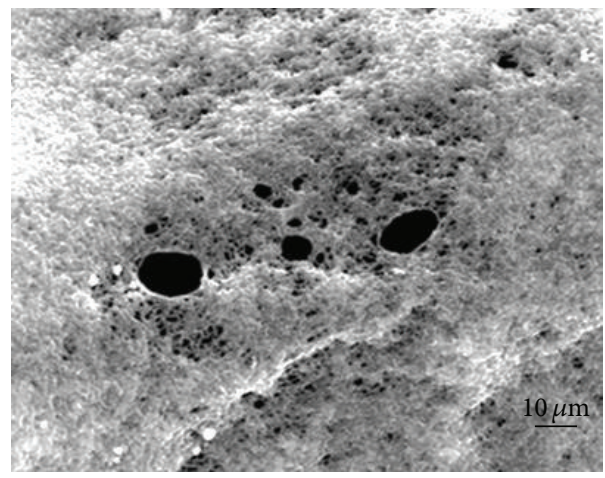

(c)

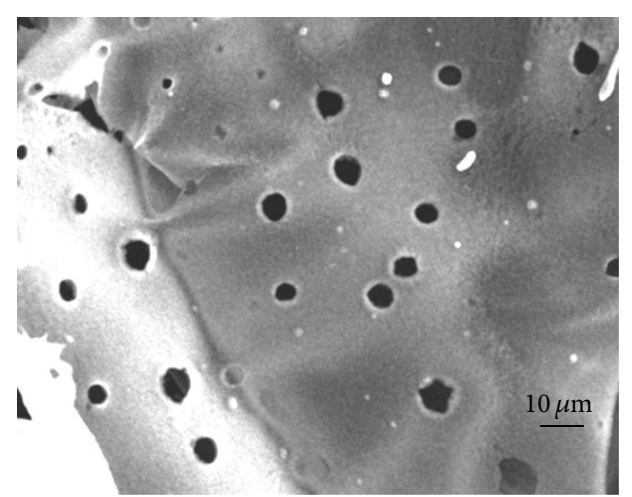

(b)

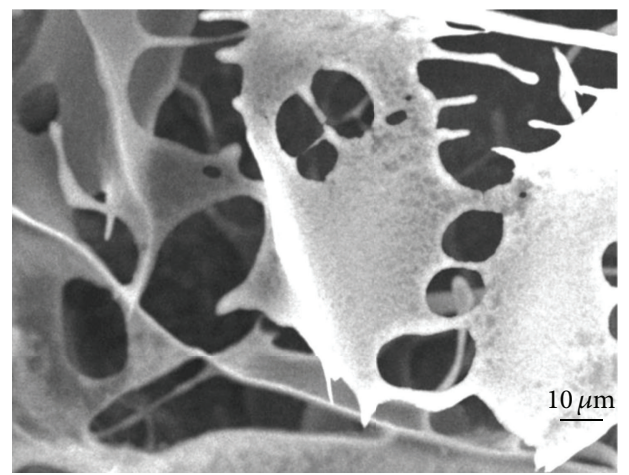

(d)

FIGURE 2: Structure of the 3D scaffold type biocomposites. FE-SEM analysis showed that the microporous structures of AL0.3/Ag0 (a) and AL0.3/Ag5 (b) had spherical pores with smooth surface; AL0.1/Ag5 (c), round shaped pores in spongy-like surface. Cross section of AL0.3/Ag5 showed microfibril edges $(\mathrm{d})$.

of artificial tissues and skin [19]. The formation of fibrillike structures is a characteristic feature for carbohydrate matrices in general. The rigidity and stability of $0.1 \%$ alginate biocomposites were lower than those of $0.3 \%$ alginate biocomposites. Due to these characteristics as well as their physical properties, the biocomposites of $0.3 \%$ alginate were analyzed further.

The elemental signals for Ag were detected in AL0.3/Ag5 but not in AL0.3/Ag0, when they were analyzed by EDS (Figure 3). The elemental signals of $\mathrm{C}, \mathrm{O}, \mathrm{Na}$, and $\mathrm{Ag}$ in the biocomposites varied in percentage, depending on the concentrations of alginate and $\mathrm{AgNO}_{3}$ used. The percentage of $\mathrm{C}, \mathrm{O}, \mathrm{Na}$, and $\mathrm{Ag}$ in $\mathrm{AL} 0.3 / \mathrm{Ag} 5$ was $51.02 \%, 40.89 \%$, $5.11 \%$, and $2.98 \%$, respectively, whereas the percentage was $54.95 \%, 39.11 \%, 5.94 \%$, and $0 \%$, respectively, in AL0.3/Ag0. The element signals, percentage, atomic weight, and series of the biocomposites were listed in the insight table of Figure 3.

The size and distribution of AgNPs in the matrix of alginate were assessed by TEM analysis (Figure 4). The nanoparticles in all samples were spherical. The size and distribution of the nanoparticles were dependent on the concentration of $\mathrm{AgNO}_{3}$, the precursor, and the alginate matrix. The mean diameter of the nanoparticles in AL0.3/Ag1, AL0.3/Ag3, and AL0.3/Ag5 was $10.0 \pm 3.3,12.0 \pm 4.0$, and $14.6 \pm 6.4 \mathrm{~nm}$, respectively (Figures $4(\mathrm{a})-4(\mathrm{c})$ ). The size of nanoparticles in $0.3 \%$ alginate biocomposite was smaller than that in $0.1 \%$ alginate biocomposite. The size of nanoparticles became smaller as the concentration of alginate increased with a fixed concentration of the precursor (data not shown), while it became larger as the concentration of the precursor increased with a fixed concentration of the matrix. It has been reported that polymers might act as capping agents for the stabilization of AgNPs [4]. The size and shape of the nanoparticles are important factors for the surface chemistry and antimicrobial property [20]. A more uniform and widely distributed (5-40 nm) size of AgNPs could be obtained when higher amount of $\mathrm{AgNO}_{3}$ (AL0.3/Ag5) was used as shown in Figure 4(d). Therefore, the micropores size of the matrix and that of nanoparticles in the matrix could be modulated by controlling the concentrations of the matrix and the precursor using SPP.

\subsection{Efficacy of AL/AgNPs Biocomposites as Antibacterial} Agents. First, the effect of AL/AgNP biocomposites on the growth of various pathogenic bacteria as antibacterial agents was tested by the agar diffusion method using discs of the 3D scaffolds (Figure 5). Generally, the growth of all the pathogens tested was affected most significantly by the AL0.3/Ag5 disc. The zone of inhibition against $E$. coli was $10.2 \mathrm{~mm}$; P. aeruginosa, $15.3 \mathrm{~mm}$ (Figures 5(a) and 5(b)); P. stutzeri, $15.7 \mathrm{~mm}$; V. parahaemolyticus, $7.6 \mathrm{~mm}$; V. vulnificus, $7.9 \mathrm{~mm}$. Among these Gram-negative bacteria, Pseudomonas strains were affected 

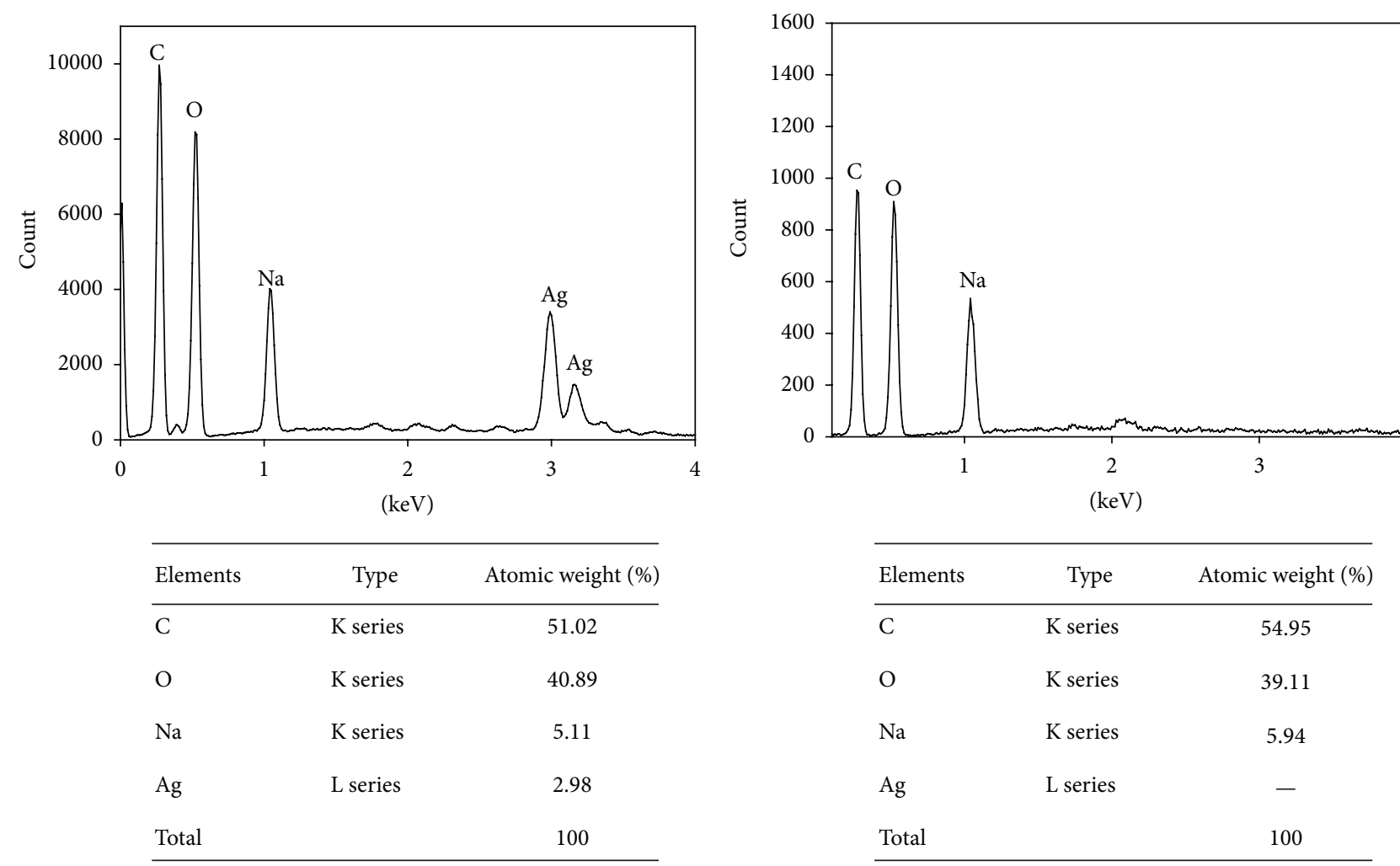

\begin{tabular}{lcc}
\hline Elements & Type & Atomic weight (\%) \\
\hline $\mathrm{C}$ & K series & 51.02 \\
$\mathrm{O}$ & K series & 40.89 \\
$\mathrm{Na}$ & K series & 5.11 \\
$\mathrm{Ag}$ & L series & 2.98 \\
Total & & 100 \\
\hline
\end{tabular}

(a)

(b)

FIGURE 3: EDS analysis of the AL0.3/Ag5 (a) and AL0.3/Ag0 (b) biocomposites. The elements, percentage, atomic weight, and series of the biocomposites are listed in the inset table.

most significantly, while Vibrio strains were affected much less than others (Figure 5, Table 1). The biocomposites affected Gram-positive bacteria less effectively than Gram-negative bacteria. The zone of growth inhibition against $S$. aureus and $B$. cereus was 11.3 and $12.6 \mathrm{~mm}$, respectively (Figures $5(\mathrm{c}$ ) and 5(d)). The varying effect of antimicrobial activity of the biocomposites against two types of bacteria was likely to be due to the difference in the structure and composition of cell wall including surface charge, cell physiology, and contact area of surface. Gram-positive bacteria have thicker and more stable cell wall than Gram-negative bacteria do. The AgNPs were likely to exert their antibacterial activity by generating reactive oxygen species. They would also disturb the signaling pathway inside the bacterial cells by modulating phosphorylation of the tyrosine residues in proteins that are important for cell viability [21].

The antibacterial activity of the AL/AgNP biocomposites was quantitatively examined by determining the least amount of the biocomposites (MIC) that was required to inhibit the growth of the bacterial pathogens in liquid culture (Table 2). MIC for the Gram-negative bacteria was $14.4 \mu \mathrm{g} / \mathrm{mL}$ for E. coli, V. parahaemolyticus, and P. stutzeri and $9.6 \mu \mathrm{g} / \mathrm{mL}$ for $V$. vulnificus. However, the MIC for P. aeruginosa was the same as that for Gram-positive bacteria, S. aureus and B. cereus $(21.0 \mu \mathrm{g} / \mathrm{mL})$. In the previous study, MIC of a gelatin/AgNP biocomposite (G3Ag5) was $20.0 \mu \mathrm{g} / \mathrm{mL}$ for E. coli and $40.0 \mu \mathrm{g} / \mathrm{mL}$ for $S$. aureus. This suggested that
AL0.3/Ag5 was more effective than G3Ag5 as an antibacterial agent [4].

3.4. Antifungal Activity of the AL/AgNP Biocomposites. Bacteria have been known to be more sensitive to AgNPs than eukaryotic microorganisms are, probably due to the difference in the cell wall structure and composition. If AgNP biocomposites affect eukaryotic microorganisms as well as bacteria (prokaryotes), they might be toxic to human cells also and inappropriate for medical or pharmaceutical purposes. Zones of growth inhibition were $6.0-7.0 \mathrm{~mm}$ for $C$. albicans and 5.0-6.0 $\mathrm{mm}$ for A. parasiticus by the AL0.3/Ag1, AL0.3/Ag3, and AL0.3/Ag5 biocomposites, suggesting that their growth was not affected significantly by the discs (Figures 5(e) and 5(f)). However, the color of the A. parasiticus spores around the AL/AgNP biocomposites remained yellow, different from other parts of the plates. Normally, mature sporangia of $A$. parasiticus would exhibit green color as the sporulation process was completed. The yellow sporangia around the biocomposite discs implied that they could not undergo complete sporulation due to the presence of AgNPs. From the results, the AL/AgNP biocomposites would not affect the growth of fungi as effectively as those of bacteria, but somehow they could partially impair sporulation of fungi.

MIC of AL/AgNPs was also examined for both fungi (Table 2). For C. albicans, MIC was determined based on the turbidity of YPD broth that was inoculated with the fresh 


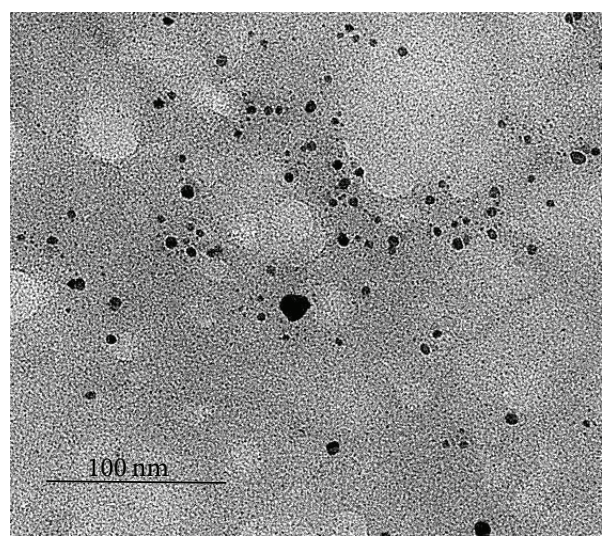

(a)

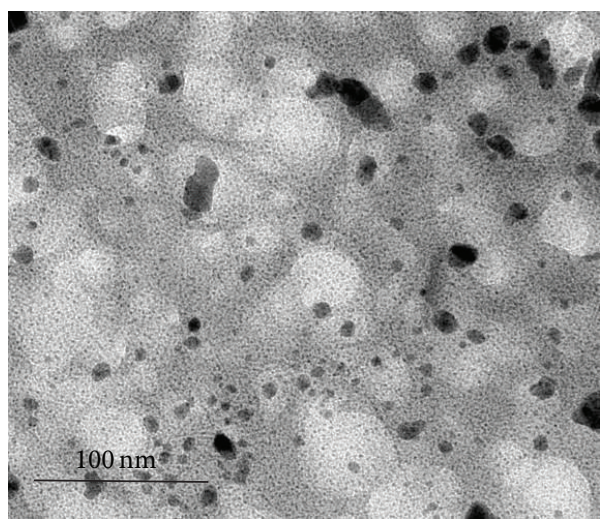

(c)

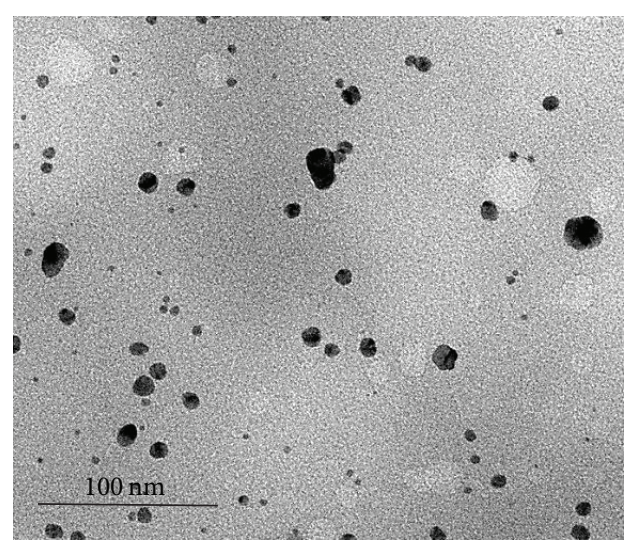

(b)

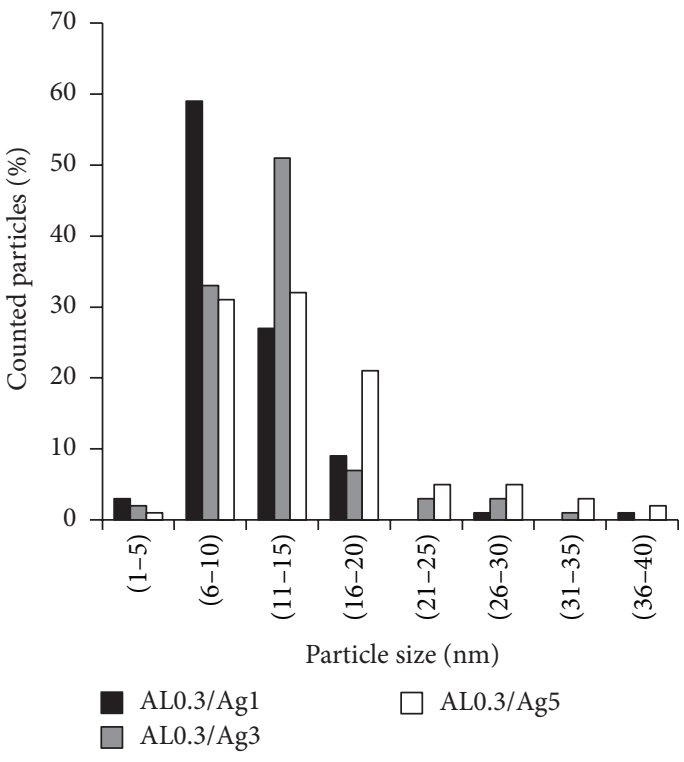

(d)

FiguRE 4: TEM analysis of the AgNPs in the biocomposites. The size and shape of the AgNPs in the biocomposites were observed after the removal of alginate. The AL0.3/Ag1 biocomposite had spherical particles with an average size of $10.0 \pm 3.3 \mathrm{~nm}(\mathrm{a}) ; \mathrm{AL} 0.3 / \mathrm{Ag} 3,12.0 \pm 4.0 \mathrm{~nm}$ (b); and AL0.3/Ag5, $14.6 \pm 6.4 \mathrm{~nm}$ (c). The graph represents the size distribution of the particles in the biocomposites (d).

yeast culture and various amounts of the AL0.3/Ag5 biocomposite. The test tubes with $85 \mu \mathrm{g} / \mathrm{mL}$ or more of AgNPs showed no turbidity due to the growth of $C$. albicans. Therefore, MIC for C. albicans was determined as $85 \mu \mathrm{g} / \mathrm{mL}$. For A. parasiticus, the fungal spores and various concentrations of the AL0.3/Ag5 biocomposite were added to PD broth in test tubes up to $510 \mu \mathrm{g} / \mathrm{mL}$. They were incubated at $25^{\circ} \mathrm{C}$ for $120 \mathrm{~h}$ before MIC was determined. However, most of the tubes took very dark color due to the large amount of the AL/AgNP biocomposite added to them and thereby assessing the hyphal growth of the fungus in the broth was impossible. Therefore, each culture was filtered through a nitrous cellulose membrane, which was then incubated on a PDA plate at $25^{\circ} \mathrm{C}$ for several days. No growth of hyphae or spore was observed on the membranes with the filtrate of the culture tubes containing $425 \mu \mathrm{g} / \mathrm{mL}$ or more of AL0.3/Ag5 and MIC of the biocomposite was determined as $425 \mu \mathrm{g} / \mathrm{mL}$. MIC against C. albicans and A. parasiticus was 6 and 30 times higher, respectively, than that against E. coli, a Gram-negative bacterium. The cell walls of yeast and filamentous fungus are much thicker and more rigid than that of bacteria. In the previous study, a gelatin/AgNP biocomposite (G3Ag5) showed MIC of $80 \mu \mathrm{g} / \mathrm{mL}$ for C. albicans and $255 \mu \mathrm{g} / \mathrm{mL}$ for $A$. parasiticus [16], indicating that the AL/AgNP biocomposite would be safer in medical application than the gelatin based AgNP biocomposite would be. AgNPs prepared by the chemical reduction method were evaluated to have considerable antifungal activity against yeast in comparison with other antifungal drugs and further investigation was suggested for clinical applications [22].

3.5. Microbicidal Activity of the AL/AgNP Biocomposites. Both the zone of growth inhibition test and the MIC test did not address whether the biocomposites exerted the antimicrobial activity by retarding the growth of or by killing the pathogens. As an effort to elucidate this, the microbicidal 
TABLE 1

\begin{tabular}{lcccc}
\hline Strains & & \multicolumn{2}{c}{ Zone of inhibition (mm) } \\
& AL0.3/Ag0 & AL0.3/Ag1 & AL0.3/Ag3 & 10.0 \\
E. coli & 5 & 9.8 & 15.2 & 10.2 \\
P. aeruginosa & 5 & 15.2 & 15.7 & 15.3 \\
P. stutzeri & 5 & 15.9 & 8.0 & 7.6 \\
V. vulnificus & 5 & 7.7 & 10.9 & 7.9 \\
V. parahaemolyticus & 5 & 7.9 & 12.2 & 11.3 \\
S. aureus & 5 & 10.6 & 6.7 & 12.6 \\
B. cereus & 5 & 12.1 & 6.0 & 7.0 \\
C. albicans & 5 & 6.0 & 6.0 \\
A. parasiticus & 5 & 5.0 & & \\
\hline
\end{tabular}

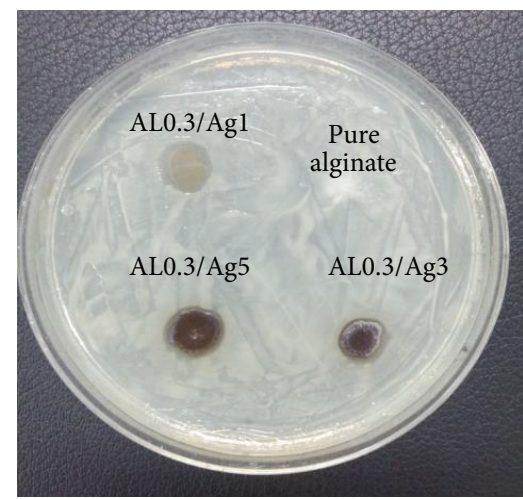

(a)

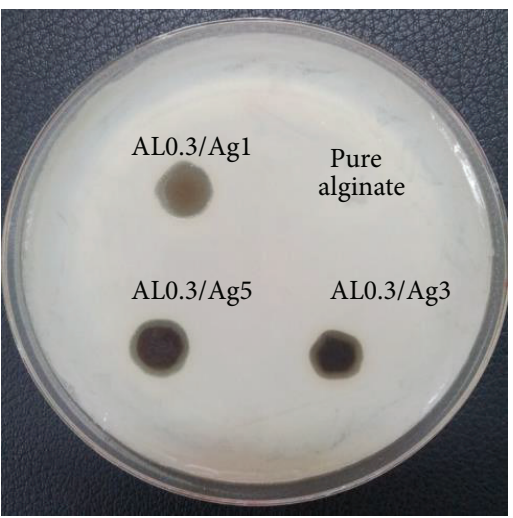

(d)

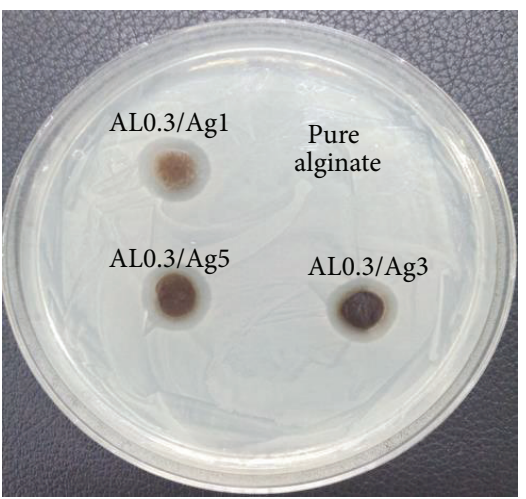

(b)

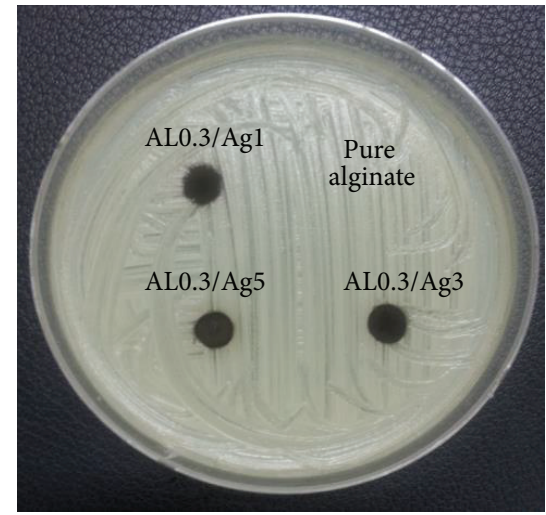

(e)

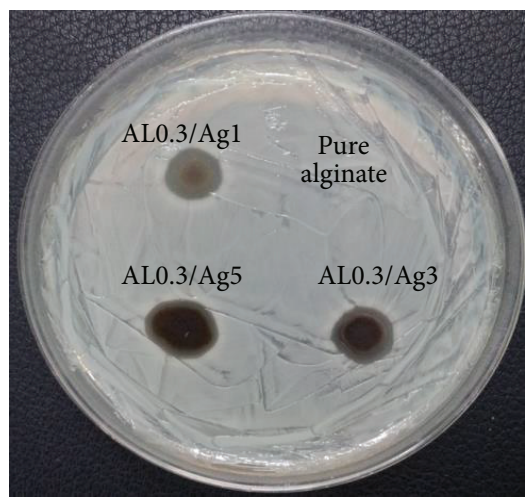

(c)

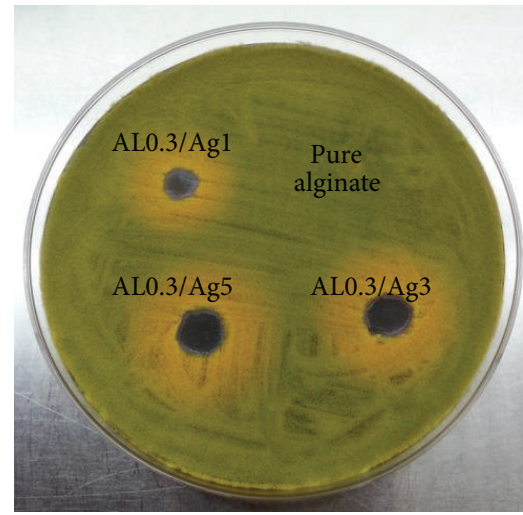

(f)

Figure 5: Agar diffusion assay of alginate/AgNPs against various pathogens. (a) E. coli, (b) P. aeruginosa, (c) S. aureus, (d) B. cereus, (e) C. albicans, and (f) A. parasiticus. The sizes of the inhibition zone formed by the biocomposites were listed in Table 1.

activity of the AL/AgNP biocomposite was investigated by quantifying the viable cells of the pathogens in CFU that survived, which were treated with various amounts of AL/AgNP biocomposites at room temperature for $1 \mathrm{~h}$. Decrease in CFU by the treatment would imply a killing effect of the biocomposite on the pathogens. Only $46.3 \%, 1.4 \%$, and $0.2 \%$ of the E. coli cells survived the treatment with AL0.3/Ag1, AL0.3/Ag3, and AL0.3/Ag5, respectively (Figure 6). The results indicated that the $E$. coli cells could be killed easily by the biocomposite, especially by AL0.3/Ag5 containing $42.5 \mu \mathrm{g} / \mathrm{mL}$ of AgNPs. Other Gram-negative bacteria were also killed as effectively as $E$. coli by the biocomposites (data not shown). Particularly, $100 \%$ of the V. vulnificus cells were killed by all 3 types of biocomposites. Among Gramnegative bacteria, $P$. aeruginosa was most resistant to the biocomposites and $37.1 \%$ of the cells survived the treatment with AL0.3/Ag5. This correlated well with the MIC data for $P$. aeruginosa, having the same MIC as those for the Gram-positive bacteria. Both S. aureus and C. albicans were resistant to the biocomposites and $88 \%$ and $73.2 \%$ of the cells, respectively, survived the treatment with AL0.3/Ag5. The survival rates of $C$. albicans were similar to those of 
TABLE 2: MIC of AL/AgNPs against various pathogens.

\begin{tabular}{lc}
\hline Pathogens & MIC $(\mu \mathrm{g} / \mathrm{mL})$ \\
\hline E. coli & 14.4 \\
P. aeruginosa & 21.0 \\
P. stutzeri & 14.4 \\
V. vulnificus & 9.6 \\
V. parahaemolyticus & 14.4 \\
S. aureus & 21.0 \\
B. cereus & 21.0 \\
C. albicans & 85.0 \\
A. parasiticus & 425.0 \\
\hline
\end{tabular}

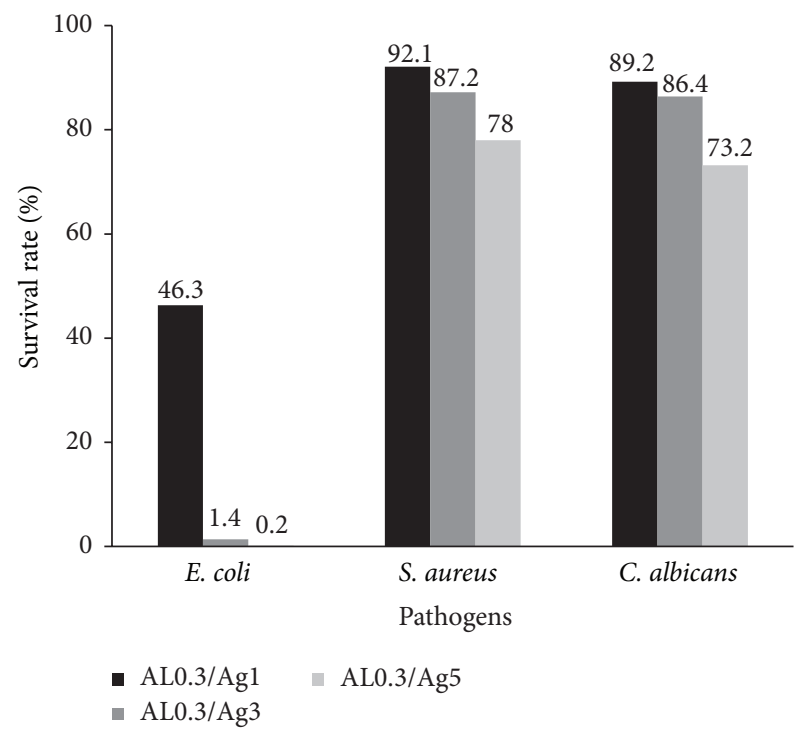

FIGURE 6: CFU reduction of the pathogens by the AL/AgNP biocomposites. CFU reductions as a function of the AgNP concentration exhibited decrease in the viable cells as the AgNP concentration increased.

S. aureus. This could be due to much fewer C. albicans cells (3fold) used for the tests. In the previous study, a gelatin/AgNP biocomposite (G3Ag5) reduced the CFU of E. coli by $100 \%$ and that of $S$. aureus by $96.3 \%$ when the bacteria were treated at $37^{\circ} \mathrm{C}$ for $1 \mathrm{~h}[4]$. It took $2 \mathrm{~h}$ for cellulose/AgNP biocomposites to kill $100 \%$ of the E. coli cells, while it took the biocomposites $8-16 \mathrm{~h}$ for completely killing $S$. aureus [5]. The results showed that the biocomposites indeed killed the pathogens and that the efficacy could be modulated by the amount of AgNPs and the treatment time, according to the target pathogen. Based on the results obtained in this study, the biocomposites of AL/AgNPs synthesized by using SPP had microbicidal activity against various pathogens and have the potential in biomedical application such as development of wound dressing materials once confirming their biocompatibility.

\section{Conclusions}

The AL/AgNP biocomposites were readily synthesized with varying concentrations of alginate and $\mathrm{AgNO}_{3}$ by the ecofriendly SPP method. AgNPs were synthesized and incorporated simultaneously into the alginate matrix by discharging plasma for $7 \mathrm{~min}$ in the solution without adding hazardous chemicals as reducing or stabilizing agents. The significance of the AL/AgNP biocomposites was that they were synthesized only using alginate in less than one-tenth amount $(0.1,0.3 \%)$ of other polymers that had been previously used as the matrix. 3D scaffolds of the biocomposites had microporous structure with spongy or smooth surface with microfibril edges. Spherical nanoparticles with a size range of 5-40 $\pm 2.0 \mathrm{~nm}$ were well distributed in the matrix. The porosity and size of the nanoparticles in the biocomposites were likely to be tuned by the concentrations of alginate and $\mathrm{AgNO}_{3}$. The AL/AgNP biocomposites exhibited a broad spectrum of microbicidal activity against various pathogens, being most effective against Gram-negative bacteria, and then in the order of Gram-positive bacteria, C. albicans, and A. parasiticus. They could inhibit the bacterial growth at much lower concentrations than those of other biocomposites. The two fungi were quite resistant to the nanobiocomposites, implying that they might be nontoxic to human cells at the concentrations for bactericidal activity and thus safe for medical application. The results suggested that the AL/AgNP biocomposites might have potential for application as wound dressing materials.

\section{Conflict of Interests}

All authors declare that they have agreed to publish this paper and that there is no content with conflict of interests.

\section{Acknowledgment}

This work was carried out by the grant from Incheon National University 2013 and the authors appreciated the financial support.

\section{References}

[1] O. Takai, "Solution plasma processing (SPP)," Pure and Applied Chemistry, vol. 80, no. 9, pp. 2003-2011, 2008.

[2] K.-H. Tseng, Y.-C. Chen, and J.-J. Shyue, "Continuous synthesis of colloidal silver nanoparticles by electrochemical discharge in aqueous solutions," Journal of Nanoparticle Research, vol. 13, no. 5, pp. 1865-1872, 2011.

[3] S. M. Kim, S. Y. Lee, M. H. Lee, and J. W. Kim, “The synthesis of $\mathrm{Pt} / \mathrm{Ag}$ bimetallic nanoparticles using a successive solution plasma process," Journal of Nanoscience and Nanotechnology, vol. 14, no. 12, pp. 8925-8929, 2014.

[4] S. C. Kim, S. M. Kim, G. J. Yoon, S. W. Nam, S. Y. Lee, and J. W. Kim, "Gelatin-based sponge with Ag nanoparticles prepared by solution plasma: fabrication, characteristics, and their bactericidal effect," Current Applied Physics, vol. 14, no. 2, pp. S172-S179, 2014. 
[5] M. Davoodbasha, S. Lee, S. Kim, and J. Kim, "One-step synthesis of cellulose/silver nanobiocomposites using a solution plasma process and characterization of their broad spectrum antimicrobial efficacy," RSC Advances, vol. 5, no. 44, pp. 35052-35060, 2015.

[6] W.-R. Li, X.-B. Xie, Q.-S. Shi, H.-Y. Zeng, Y.-S. Ou-Yang, and Y.-B. Chen, "Antibacterial activity and mechanism of silver nanoparticles on Escherichia coli," Applied Microbiology and Biotechnology, vol. 85, no. 4, pp. 1115-1122, 2010.

[7] N. Saito, J. Hieda, and O. Takai, "Synthesis process of gold nanoparticles in solution plasma," Thin Solid Films, vol. 518, no. 3, pp. 912-917, 2009.

[8] Z. S. Pillai and P. V. Kamat, "What factors control the size and shape of silver nanoparticles in the citrate ion reduction method?" The Journal of Physical Chemistry B, vol. 108, no. 3, pp. 945-951, 2004.

[9] K. C. Song, S. M. Lee, T. S. Park, and B. S. Lee, "Preparation of colloidal silver nanoparticles by chemical reduction method," Korean Journal of Chemical Engineering, vol. 26, no. 1, pp. 153155, 2009.

[10] D. MubarakAli, J. ArunKumar, M. Rahuman Sheriff, D. Pandiaraj, K. A. ShiekSyedIshack, and N. Thajuddin, "Fabrication of silver nanoparticles with cotton for antibacterial wound dressing," Pharmaceutical Nanotechnology, vol. 1, no. 1, pp. 7882, 2013.

[11] H. Grasdalen, "High-field, ${ }^{1}$ H-n.m.r. spectroscopy of alginate: sequential structure and linkage conformations," Carbohydrate Research, vol. 118, pp. 255-260, 1983.

[12] E. Broderick, H. Lyons, T. Pembroke, H. Byrne, B. Murray, and M. Hall, "The characterisation of a novel, covalently modified, amphiphilic alginate derivative, which retains gelling and nontoxic properties," Journal of Colloid and Interface Science, vol. 298, no. 1, pp. 154-161, 2006.

[13] H. H. Tønnesen and J. Karlsen, "Alginate in drug delivery systems," Drug Development and Industrial Pharmacy, vol. 28, no. 6, pp. 621-630, 2002.

[14] S. K. Balavandy, K. Shameli, and Z. Z. Abidin, "Rapid and green synthesis of silver nanoparticles via sodium alginate media," International Journal of Electrochemical Science, vol. 10, no. 1, pp. 486-497, 2015.

[15] A. Watthanaphanit, G. Panomsuwan, and N. Saito, "A novel one-step synthesis of gold nanoparticles in an alginate gel matrix by solution plasma sputtering," RSC Advances, vol. 4, no. 4, pp. 1622-1629, 2014.

[16] S.-C. Kim, J.-W. Kim, G.-J. Yoon, S.-W. Nam, and S.-Y. Lee, "Antifungal effects of 3D scaffold type gelatin/Ag nanoparticles biocomposite prepared by solution plasma processing," Current Applied Physics, vol. 13, supplement 1, pp. S48-S53, 2013.

[17] A. W. Bauer, W. M. Kirby, J. C. Sherris, and M. Turck, "Antibiotic susceptibility testing by a standardized single disk method," The American Journal of Clinical Pathology, vol. 45, no. 4, pp. 493496, 1966.

[18] S. H. Jin, S. M. Kim, S. Y. Lee, and J. W. Kim, "Synthesis and characterization of silver nanoparticles using a solution plasma process," Journal of Nanoscience and Nanotechnology, vol. 14, no. 10, pp. 8094-8097, 2014.

[19] C. J. Grande, F. G. Torres, C. M. Gomez, and M. C. Bañó, "Nanocomposites of bacterial cellulose/hydroxyapatite for biomedical applications," Acta Biomaterialia, vol. 5, no. 5, pp. 1605-1615, 2009.
[20] M. Rai, A. Yadav, and A. Gade, "Silver nanoparticles as a new generation of antimicrobials," Biotechnology Advances, vol. 27, no. 1, pp. 76-83, 2009.

[21] V. Gopinath and P. Velusamy, "Extracellular biosynthesis of silver nanoparticles using Bacillus sp. GP-23 and evaluation of their antifungal activity towards Fusarium oxysporum," Spectrochimica Acta Part A: Molecular and Biomolecular Spectroscopy, vol. 106, pp. 170-174, 2013.

[22] A. Nasrollahi, K. H. Pourshamsian, and P. Mansourkiaee, "Antifungal activity of silver nanoparticles on some of fungi," International Journal of Nano Dimension, vol. 1, no. 3, pp. 233239, 2011. 

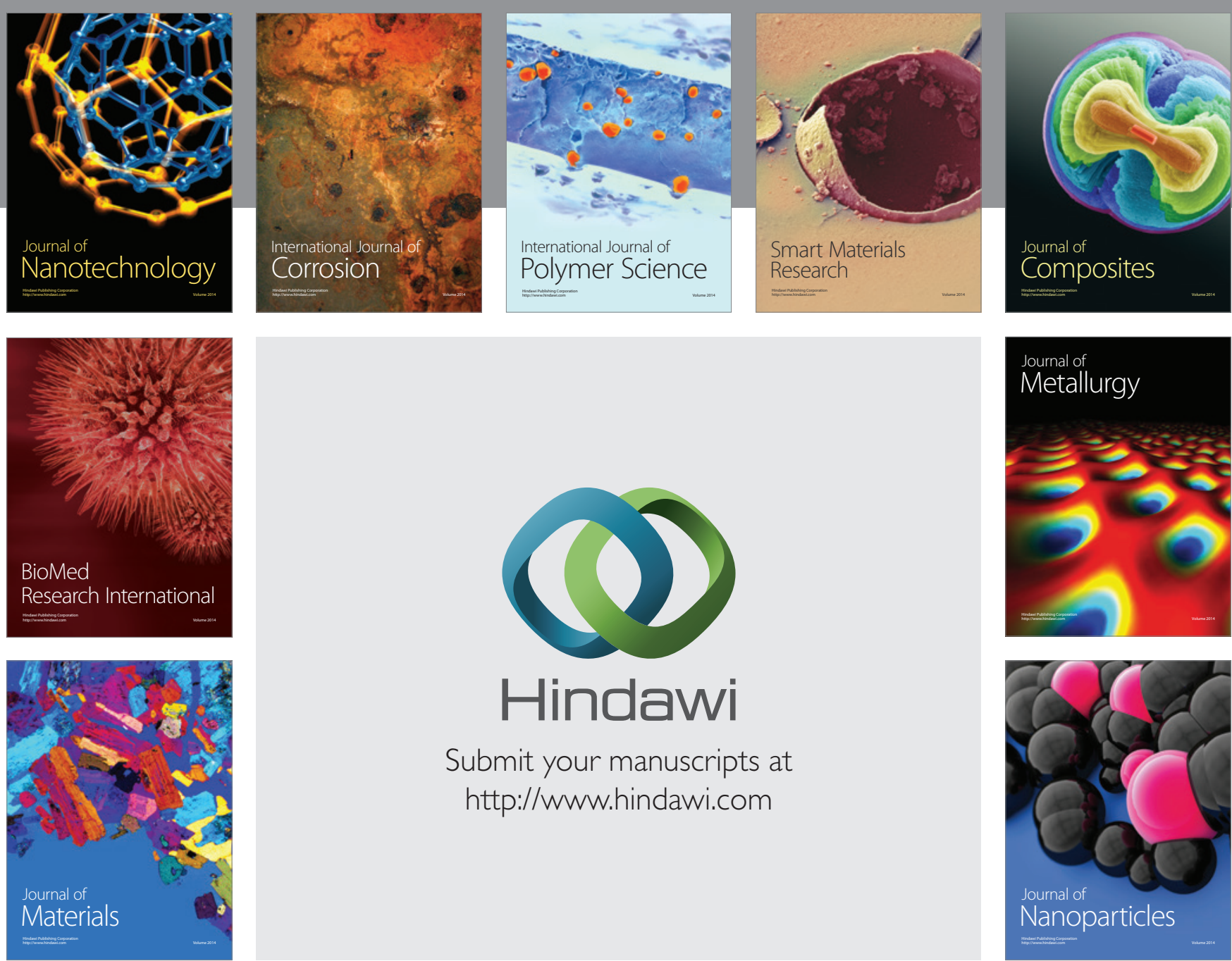

\section{Hindawi}

Submit your manuscripts at

http://www.hindawi.com

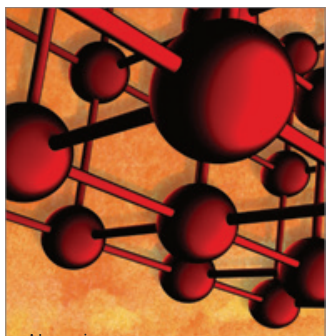

Materials Science and Engineering
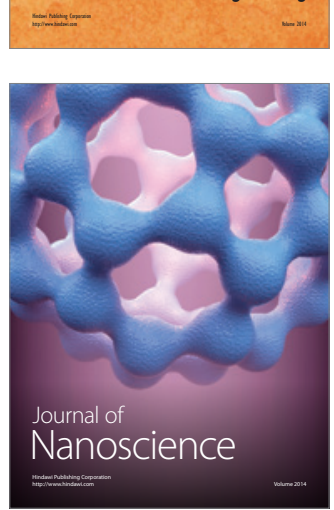
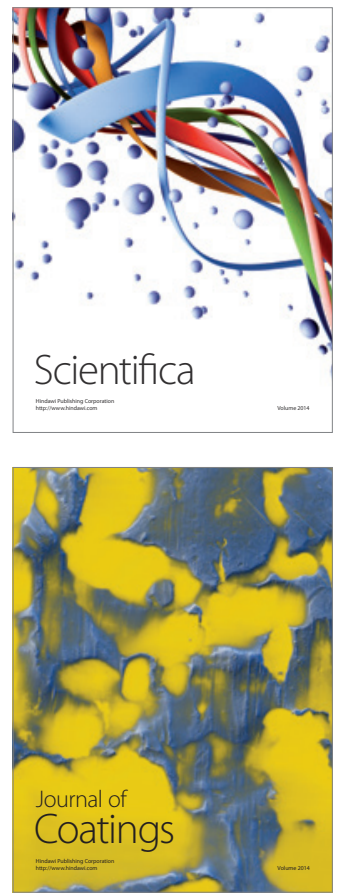
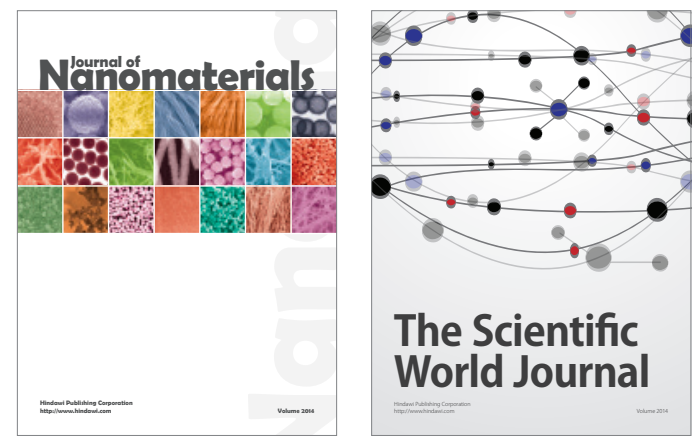

The Scientific World Journal
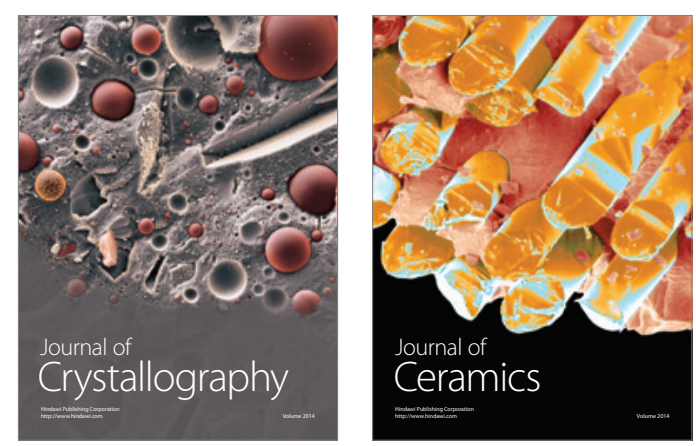
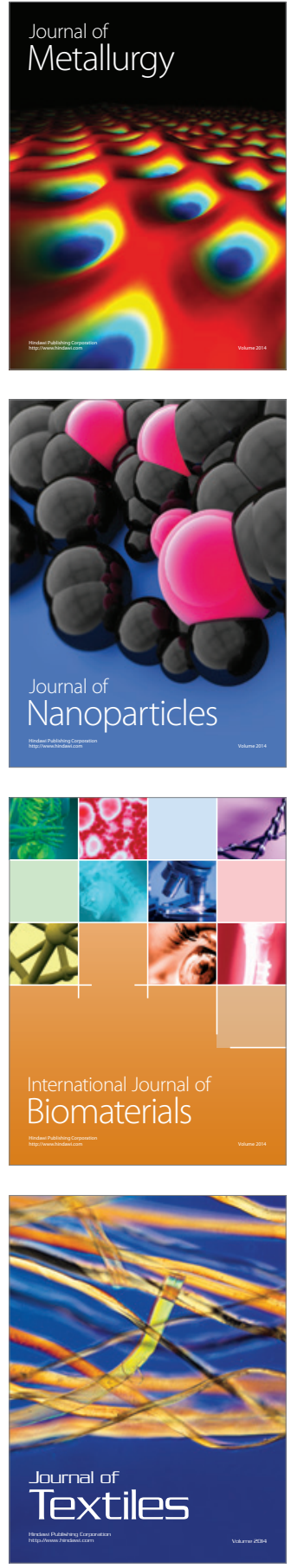